\title{
Potencial discriminatório de três testadores em "topcrosses" de milho(1)
}

\author{
Iran de Azevedo Duarte ${ }^{(2)}$, Josué Maldonado Ferreira ${ }^{(2)}$ e Cláudio Natalino Nuss ${ }^{(3)}$
}

Resumo - O objetivo deste trabalho foi determinar o potencial de três testadores endogâmicos elites a fim de avaliar e classificar 15 materiais genéticos para a síntese de híbridos. Quarenta e cinco híbridos mais quatro testemunhas foram avaliados em cinco ambientes, no ano agrícola 2000/2001, utilizando delineamento em blocos ao acaso, com três repetições. Foram avaliados os caracteres peso de grãos corrigido, altura de planta e altura de espiga. As estimativas de capacidade geral e específica de combinação foram obtidas pelo modelo de Griffing, adaptado por Vencovsky e Barriga, para análise em delineamento genético fatorial. O rendimento médio obtido entre os cinco ambientes variou de $8,97 \mathrm{t} \mathrm{ha}^{-1}$ a $12,21 \mathrm{t} \mathrm{ha}^{-1}$. Vinte e sete tratamentos superaram a média geral absoluta da melhor testemunha. A análise de variância conjunta revelou efeitos significativos de ambientes, capacidade geral de combinação dos parentais e testadores, capacidade específica de combinação e das interações ambiente x capacidade geral de combinação dos parentais e testadores. Os testadores promoveram uma classificação diferenciada para a base genética avaliada, com destaque para os cruzamentos entre parentais HS1, HS2, HS5 e HT4 com o testador LF e parentais L10 e HS5 com o testador L05.

Termos para indexação: Zea mays, progênie, híbrido, capacidade combinatória, germoplasma.

\section{Screening potential of three maize topcross testers}

\begin{abstract}
The objective of this work was to assess the potential of three elite inbred testers to evaluate and rank fifteen genetic materials for hybrid synthesis. Forty-five hybrids and four controls were evaluated in five different environments in the 2000/2001 growing season using a randomized complete block design with three replications. The main agronomic traits were assessed, including the corrected grain yield and plant and ear height. Estimates of general and specific combining ability were obtained using Griffing's method adapted by Vencovsky and Barriga. The average yield in the five environments ranged from 8.97 ton ha ${ }^{-1}$ to 12.21 ton $\mathrm{ha}^{-1}$. Twenty seven treatments outperformed the mean yield of the best control. The analysis of variance over environments revealed significant main effects of environments, general combining ability of the parents and testers, specific combining ability and significant interactions, such as environment $\mathrm{x}$ general combining ability of parents and also environment $\mathrm{x}$ general combining ability of testers. The testers allowed ranking and screening of the assessed genetic basis, suggesting as best combinations the crosses that included the HS1, HS2, HS5 and HT4 parents with the LF tester and the L10 and HS5 parents with the L05 tester.
\end{abstract}

Index terms: Zea mays, progeny, hybrids, combining ability, germplasm.

(1) Aceito para publicação em 5 de dezembro de 2002.

Extraído da dissertação de mestrado, apresentada pelo primeiro autor à Universidade Estadual de Londrina (UEL). Financiado pela Capes.

(2)UEL, Dep. de Ciências Biológicas, Caixa Postal 6001, CEP 86051-990 Londrina, PR.

E-mail: iranduarte@hotmail.com,josuemf@uel.br

(3) Agroeste Sementes Ltda., Caixa Postal 185, CEP 89820-000

Xanxerê, SC. E-mail: agroeste@agroeste.com.br

\section{Introdução}

A seleção de testadores é um dos processos mais importantes na escolha de linhagens de milho para a identificação de combinações híbridas superiores.

Geralmente, as melhores combinações híbridas estão diretamente relacionadas com o grau de divergência genética dos parentais envolvidos (Ferreira 
et al., 1995; Hallauer \& Miranda Filho, 1995; Sinobas \& Monteagudo, 1996; Magnavaca, 1998). Porém, esta divergência não tem determinado necessariamente a máxima expressão da heterose (Dudley et al., 1992; Vasal et al., 1992b). Assim, a seleção de genitores e a utilização de métodos que identifiquem as melhores combinações são etapas de grande importância.

Entre os métodos disponíveis para avaliar a capacidade combinatória (CC), destacam-se, pela grande utilização, os esquemas de "topcrosses" (Radovic \& Jelovac, 1995; Eyhérabide \& Gonzalez, 1997; Santos et al., 2001) e os cruzamentos dialélicos (Sinobas \& Monteagudo, 1996; San Vicente et al., 1998; Pinto et al., 2001). Esquemas de "topcrosses" têm sido adotados, principalmente pela facilidade de execução e obtenção dos parâmetros de capacidade de combinação. Neste esquema, a CC é determinada pelo cruzamento entre um ou mais testadores, previamente selecionados, com um grupo de genótipos a serem avaliados. A seleção do testador ideal irá depender dos objetivos de cada programa, podendo estar fundamentada na alta ou baixa freqüência de alelos favoráveis, base genética ampla ou estreita, capacidade geral ou específica de combinação, rendimento per se, número de testadores utilizados e grau de parentesco com os materiais avaliados (Hallauer \& Miranda Filho, 1995; Castellanos et al., 1998; Santos et al., 2001). Um bom testador, para qualquer programa de desenvolvimento de híbridos, deve oferecer simplicidade no uso, gerar informação que classifique corretamente o potencial relativo das linhagens em cruzamento e maximizar o ganho genético.

Como os programas de melhoramento vêm direcionando seus objetivos para a obtenção de híbridos de alto desempenho, enquadrando-se nesta categoria híbridos simples, simples modificados e triplos, a grande maioria dos testadores utilizados têm sido constituída por linhagens elites, fornecendo a possibilidade de obter materiais com potencial de mercado, além de proporcionar a predição do comportamento de possíveis híbridos triplos e duplos.

O objetivo deste trabalho foi determinar o potencial de três testadores endogâmicos elites a fim de avaliar e classificar 15 materiais genéticos para a síntese de híbridos.

\section{Material e Métodos}

No ano agrícola 1999/2000, foi obtido o material experimental, mediante o cruzamento de três linhagens elites testadoras (LF, L09 e L05), divergentes nas suas origens, com 15 diferentes materiais genéticos parentais: duas linhagens (L06 e L10), cinco híbridos simples (HS), quatro híbridos triplos (HT), dois híbridos duplos (HD) e duas populações melhoradas (Poblacion 36 e CMS 14-C), na Estação Experimental da Empresa Agroeste Sementes Ltda., Xanxerê, SC. A síntese dos cruzamentos foi realizada em três lotes isolados e cada lote foi formado por um dos testadores utilizado como macho (polinizador) e 15 parentais como fêmeas (linhas despendoadas). A caracterização do material experimental encontra-se na Tabela 1.

O potencial dos 45 híbridos, mais quatro testemunhas (Z8392 - híbrido simples da Zeneca; P3071 - híbrido triplo da Pioneer; Poblacion 36 do CIMMYT e CMS 14-C, uma população da Embrapa-Centro Nacional de Pesquisa de Milho e Sorgo), foi avaliado no ano agrícola 2000/ 2001. Foi utilizado o delineamento de blocos ao acaso, com três repetições, em cinco ambientes (A1, A2, A3, A4 e A5), correspondendo aos ambientes de Londrina, PR $\left(23^{\circ} 23^{\prime} \mathrm{S}, 51^{\circ} 11^{\prime} \mathrm{W}\right.$ e $576 \mathrm{~m}$ de altitude), Palotina, PR $\left(24^{\circ} 12^{\prime} \mathrm{S}, 53^{\circ} 50^{\prime} \mathrm{W}\right.$ e $289 \mathrm{~m}$ de altitude), Xanxerê, SC, épocas 1 e $2\left(27^{\circ} 7^{\prime} \mathrm{S}, 52^{\circ} 37^{\prime} \mathrm{W}\right.$ e $630 \mathrm{~m}$ de altitude) e Pinhão, PR ( $25^{\circ} 41^{\prime} \mathrm{S}, 51^{\circ} 38^{\prime} \mathrm{W}$ e $1.120 \mathrm{~m}$ de altitude), respectivamente.

As parcelas foram constituídas por duas fileiras de 4,0 $\mathrm{m}$ de comprimento, com duas plantas por cova, a intervalos de 0,80x0,40 m, para garantir um estande ideal de 40 plantas por parcela e atingir uma densidade aproximada de 62.500 plantas ha ${ }^{-1}$.

As adubações de base e cobertura e o controle de pragas e plantas daninhas foram realizadas seguindo as recomendações técnicas para a cultura do milho, sob condições de alta tecnologia.

Foram avaliados os caracteres peso de grãos corrigido (PGC, em t ha ${ }^{-1}$ ) para a umidade de $13 \%$ e estande ideal, altura de planta $(\mathrm{AP}, \mathrm{em} \mathrm{cm})$ e altura de espiga $(\mathrm{AE}$, $\mathrm{em} \mathrm{cm}$ ). As correções de estande para o caráter PGC foram realizadas utilizando peso total de parcelas para o estande ideal (STi), pelo método de correção por covariância, modificado por Miranda Filho (Vencovsky \& Barriga, 1992).

Inicialmente, foram realizadas análises de variâncias individuais de médias e de totais de parcelas em cada ambiente, segundo o delineamento de blocos ao acaso. A partir das análises individuais de variância, foi realizada a análise dos "topcrosses", pelo modelo de Griffing 
(1956), adaptado por Vencovsky \& Barriga (1992) para análise em delineamento genético fatorial, calculado com base nas médias dos tratamentos:

$Y_{i j}=\mu+g_{i}+g_{j}+s_{i j}+\bar{\varepsilon}_{i j}$,

sendo $\mathrm{Y}_{\mathrm{ij}} \mathrm{o}$ valor médio da combinação híbrida entre o i-ésimo parental do grupo 1 e o j-ésimo testador do grupo $2 ; \mu$, a média geral; $\mathrm{g}_{\mathrm{i}}$, o efeito da capacidade geral de combinação do i-ésimo parental do grupo $1 ; \mathrm{g}_{\mathrm{j}}$, o efeito da capacidade geral de combinação do j-ésimo testador do grupo $2 ; \mathrm{s}_{\mathrm{ij}}$, o efeito da capacidade específica de combinação entre os parentais e os testadores de ordem i e j, dos grupos 1 e 2 , respectivamente, e $\bar{\varepsilon}_{\mathrm{ij}}$, o erro experimental médio.

A análise conjunta de variância dos caracteres avaliados foi realizada segundo o método proposto por Miranda Filho \& Vencovsky (1995), utilizando médias de tratamentos. Para esta análise, o efeito de ambiente foi considerado como aleatório. Desta forma, os efeitos principais foram comparados com a interação ambiente $\mathrm{x}$ tratamento e as demais interações comparadas com o resíduo.

\section{Resultados e Discussão}

A análise conjunta de variância revelou efeitos significativos $(\mathrm{P}<0,01$ e $\mathrm{P}<0,05)$ na maioria das fontes de variação para os três caracteres avaliados (Tabela 2). No desdobramento da fonte de variação, verificou-se efeitos significativos relacionados à capacidade geral de combinação dos parentais (CGC I), dos testadores (CGC II) e à capacidade específica de combinação (CEC), indicando um comportamento diferenciado dos parentais, testadores e cruzamentos, quanto à capacidade combinatória geral e específica.

No contraste entre "topcross" e a testemunha, a ausência de significância do caráter AP indica que os cruzamentos apresentaram altura de planta que não diferiu dos materiais de padrão comercial, enquanto a significância para PGC revelou que os cruzamentos apresentaram comportamento que diferiu das testemunhas, produzindo em média $1,0 \mathrm{t} \mathrm{ha}^{-1}$ a mais.

Tabela 1. Características agronômicas de três linhagens elites testadoras, de quinze materiais genéticos parentais utilizados na síntese dos "topcrosses" e de quatro testemunhas de milho, 1999/2000.

\begin{tabular}{llllll}
\hline Material & Tipo do grão & Cor do grão & Origem & Ciclo & Porte \\
\hline LF & & & Testadoras & \\
L09 & Duro & Amarela & Subtropical & Tardio & Médio/alto \\
L05 & Duro & Amarela & Temperado & Precoce & Baixo \\
& Dentado & Amarela & Temperado & Tardio & Alto \\
L06 & & & Parentais & \\
L10 & Duro & Laranja & Tropical & Precoce & Baixo \\
HS 1 & Dentado & Amarela & Temperado & Precoce & Baixo \\
HS 2 & Semi-duro & Laranja & Subtropical & Precoce & Baixo \\
HS 3 & Duro & Laranja & Subtropical & Precoce & Baixo \\
HS 4 & Semi-duro & Laranja & Subtropical & Precoce & Baixo \\
HS 5 & Semi-dentado & Amarela & Subtropical & Super precoce & Baixo \\
HT 1 & Duro & Laranja & Subtropical & Precoce & Médio \\
HT 2 & Duro & Vermelha & Subtropical & Super precoce & Baixo \\
HT 3 & Semi-dentado & Amarela & Subtropical & Super precoce & Médio/alto \\
HT 4 & Dentado & Amarela & Subtropical & Precoce & Médio/alto \\
HD 1 & Duro & Laranja & Subtropical & Precoce & Médio \\
HD 2 & Duro & Laranja & Subtropical & Precoce & Médio/alto \\
Pob. 36 & Semi-dentado & Amarela & Subtropical & Precoce & Alto \\
CMS 14-C & Dentado & Amarela & Tropical & Precoce & Alto \\
\hline & Dentado & Amarela & Tropical & Normal & Alto \\
Z8392 & Semi-duro & Laranja & Testemunhas & & \\
P3071 & Duro & Laranja & Subtropical & Precoce & Baixo \\
Pob. 36 & Dentado & Amarela & Tropical & Precoce & Médio \\
CMS 14-C & Dentado & Amarela & Tropical & Normal & Alto \\
\hline & & & & & Alto \\
\hline
\end{tabular}


A interação ambiente $\mathrm{x}$ tratamento foi significativa para os três caracteres, sugerindo que tanto os "topcrosses" quanto as testemunhas não apresentaram a mesma performance relativa frente a variação ambiental a que foram submetidos. Este tipo de interação requer a adoção de estratégias que aproveitem ou minimizem este efeito, mediante a divisão geográfica das áreas ou da recomendação de genótipos a condições ambientais específicas (Ramalho et al., 1993; Duarte \& Paterniani, 1998; Santos et al., 2001).

Foram verificados efeitos significativos $(\mathrm{P}<0,01)$ da interação ambiente x "topcross", ambiente x CGC I e ambiente x CGC II e não-significativos para ambiente x CEC. Trabalhos têm demonstrado que efeitos de CGC e CEC manifestam interação com o ambiente (Vasal et al., 1992a; Everett et al., 1995). Diante desta situação, Nass et al. (2000) apontaram para a necessidade de selecionar parentais dentro de ambientes específicos, como uma alternativa viável para maximizar o rendimento híbrido.

As médias gerais dos cruzamentos, obtidas entre os cinco ambientes para o caráter PGC, variaram de $8,97 \mathrm{t} \mathrm{ha}^{-1}$ (HD1 x L09) a 12,21 t ha-1 (HS5 x LF), revelando o elevado potencial produtivo destes cruzamentos (Tabela 3). A maior média geral do ensaio foi obtida no ambiente A1 $\left(12,59 \mathrm{t} \mathrm{ha}^{-1}\right)$, cuja precisão experimental $(\mathrm{CV}=4,39 \%)$ foi a melhor. A média geral absoluta, obtida pela melhor testemunha Z8392 (10,95 $\left.\mathrm{t} \mathrm{ha}^{-1}\right)$, foi superada por $27 \mathrm{cru}-$ zamentos. Os 10 cruzamentos mais produtivos atingiram a média de 11,95 t ha ${ }^{-1}$; o testador LF esteve presente nas oito melhores combinações e os efeitos positivos de CEC foram consideráveis em apenas quatro. O testador L09 não esteve presente entre os 27 cruzamentos que superaram a média geral absoluta da melhor testemunha. Estes resultados revelam que a combinação entre parentais x testador LF e parentais x testador L09 resultaram nos maiores e menores rendimentos, respectivamente.

A classificação dos cruzamentos determinada pela linhagem avaliadora em um ambiente não se manteve nos demais ambientes. Este fato pode ser atribuído à origem de cada avaliador, origem dos parentais (Tabela 1) e aos ambientes de avaliação, sugerindo a necessidade de seleção de linhagens avaliadoras para ambientes específicos. Desta forma, a utilização de apenas uma linhagem como avaliadora pode

Tabela 2. Análise de variância conjunta da avaliação de "topcrosses" em cinco ambientes, para os caracteres peso de grãos corrigidos (PGC, em t ha-1), altura de planta (AP, em cm) e altura de espiga (AE, em cm) em milho, 2000/2001.

\begin{tabular}{lrrrr}
\hline Fonte de variação & GL & \multicolumn{3}{c}{ Quadrado Médio } \\
\cline { 2 - 4 } & & \multicolumn{1}{c}{ PGC } & AP & \multicolumn{1}{c}{ AE } \\
\hline Ambientes & 4 & $53,7620^{* *}$ & $9.670,7000^{* *}$ & $7.741,6000^{* *}$ \\
Tratamentos & 48 & $3,2674^{* *}$ & $713,1300^{* *}$ & $500,5600^{* *}$ \\
"Topcross" (Top) & 44 & $2,8195^{* *}$ & $761,8300^{* *}$ & $510,7400^{* *}$ \\
$\quad$ Parentais (CGC I) & 14 & $1,8897^{* *}$ & $465,9500^{* *}$ & $284,7800^{* *}$ \\
$\quad$ Testadores (CGC II) & 2 & $40,6020^{* *}$ & $12.585,0000^{* *}$ & $8.883,8000^{* *}$ \\
$\quad$ Parental x testador (CEC) & 28 & $0,5857^{* *}$ & $65,2310^{* *}$ & $25,6470^{* *}$ \\
Testemunhas & 3 & $4,4831^{* *}$ & $235,8200^{* *}$ & $432,1700^{* *}$ \\
Top vs. testemunha & 1 & $19,3280^{* *}$ & $2,2005^{\mathrm{ns}}$ & $257,8300^{* *}$ \\
Ambiente x tratamento & 192 & $0,3183^{* *}$ & $19,4270^{* *}$ & $13,3490^{* *}$ \\
Ambiente x "topcross" & 176 & $0,3189^{* *}$ & $19,5430^{* *}$ & $12,6980^{* *}$ \\
$\quad$ Ambiente x CGC I & 56 & $0,3391^{* *}$ & $19,4440^{*}$ & $16,9960^{* *}$ \\
Ambiente x CGC II & 8 & $2,4095^{* *}$ & $109,3100^{* *}$ & $24,2530^{* *}$ \\
$\quad$ Ambiente x CEC & 112 & $0,1595^{\mathrm{ns}}$ & $13,1810^{\mathrm{ns}}$ & $9,7242^{\mathrm{ns}}$ \\
Ambiente x testemunha & 12 & $0,3327^{* *}$ & $18,2940^{\mathrm{ns}}$ & $14,7070^{\mathrm{ns}}$ \\
Ambiente x (top vs. testemunha) & 4 & $0,2456^{\mathrm{ns}}$ & $17,7200^{\mathrm{ns}}$ & $37,9250^{* *}$ \\
Resíduo & 475 & 0,1499 & $14,0600^{(1)}$ & $8,5806^{(1)}$ \\
\hline Média & & 10,97 & 220 & 120 \\
CV (\%) & 6,11 & 2,95 & 4,23 \\
\hline
\end{tabular}

${ }^{(1)} \mathrm{GL}$ do resíduo corresponde a 480 para os caracteres AP e AE. ${ }^{\text {ns }}$ Não-significativo. * e **Significativo a $5 \%$ e a $1 \%$ de probabilidade, respectivamente 
Tabela 3. Peso de grãos corrigido de "topcrosses" e testemunhas (PGC, em t ha ${ }^{-1}$ ), capacidade específica de combinação (CEC), coeficiente de variação experimental (CV) e diferença mínima significativa (DMS). Média de cinco ambientes, 2000/2001(1).

\begin{tabular}{|c|c|c|c|c|c|c|c|c|c|c|c|c|}
\hline \multirow[t]{2}{*}{ Tratamentos } & \multicolumn{2}{|c|}{ A1 } & \multicolumn{2}{|c|}{$\mathrm{A} 2$} & \multicolumn{2}{|c|}{ A3 } & \multicolumn{2}{|c|}{ A4 } & \multicolumn{2}{|c|}{ A5 } & \multicolumn{2}{|c|}{ Média } \\
\hline & PGC & $\mathrm{CEC}$ & PGC & $\mathrm{CEC}$ & PGC & CEC & PGC & CEC & PGC & CEC & PGC & CEC \\
\hline HS5xLF & 13,56 & 0,15 & 12,02 & $-0,21$ & 11,74 & 0,35 & 10,94 & $-0,06$ & 12,78 & 0,42 & 12,21 & 0,13 \\
\hline HS1xLF & 13,01 & $-0,12$ & 11,77 & $-0,24$ & 12,39 & 0,40 & 11,03 & $-0,35$ & 12,76 & 0,47 & 12,19 & 0,03 \\
\hline HT3xLF & 13,14 & $-0,19$ & 12,36 & 0,32 & 12,43 & 0,44 & 10,96 & $-0,19$ & 11,44 & $-0,54$ & 12,07 & $-0,03$ \\
\hline HS $2 x L F$ & 13,53 & $-0,41$ & 11,84 & 0,13 & 11,58 & $-0,14$ & 11,35 & 0,29 & 11,47 & $-0,06$ & 11,95 & $-0,04$ \\
\hline HT4xLF & 12,95 & 0,06 & 11,62 & 0,30 & 11,72 & 1,03 & 11,02 & 0,65 & 11,78 & 0,76 & 11,82 & 0,56 \\
\hline HD2xLF & 13,07 & 0,05 & 11,52 & $-0,21$ & 11,37 & $-0,14$ & 10,98 & $-0,46$ & 12,18 & 0,28 & 11,82 & $-0,10$ \\
\hline HS3xLF & 13,98 & 0,66 & 11,23 & 0,08 & 10,45 & $-0,21$ & 11,55 & 0,58 & 11,80 & 0,19 & 11,80 & 0,26 \\
\hline L10xLF & 12,52 & $-0,39$ & 11,55 & 0,37 & 11,61 & 0,71 & 10,98 & 0,44 & 12,02 & 0,80 & 11,74 & 0,39 \\
\hline HS5xL05 & 13,35 & 0,46 & 12,84 & 0,30 & 10,47 & $-0,23$ & 9,98 & $-0,16$ & 12,01 & $-0,27$ & 11,73 & 0,02 \\
\hline HS2xL05 & 13,38 & $-0,05$ & 12,10 & 0,06 & 11,23 & 0,20 & 10,22 & 0,02 & 11,68 & 0,22 & 11,72 & 0,09 \\
\hline HS1xL05 & 12,09 & $-0,52$ & 12,95 & 0,62 & 11,13 & $-0,18$ & 10,95 & 0,44 & 11,41 & $-0,80$ & 11,70 & $-0,09$ \\
\hline HT2xLF & 13,89 & 0,31 & 10,96 & $-0,59$ & 10,74 & $-0,33$ & 11,05 & 0,06 & 11,80 & $-0,05$ & 11,69 & $-0,12$ \\
\hline L06xLF & 13,66 & 0,19 & 12,00 & $-0,21$ & 10,57 & $-0,55$ & 9,68 & $-0,16$ & 11,89 & $-0,39$ & 11,56 & $-0,22$ \\
\hline HT3xL05 & 12,67 & $-0,14$ & 12,32 & $-0,04$ & 10,88 & $-0,42$ & 9,77 & $-0,51$ & 11,90 & 0,00 & 11,51 & $-0,22$ \\
\hline HT2xL05 & 13,14 & 0,07 & 11,71 & $-0,16$ & 10,78 & 0,39 & 9,98 & $-0,13$ & 11,82 & 0,05 & 11,49 & 0,04 \\
\hline HS4xLF & 12,96 & $-0,03$ & 11,33 & 0,08 & 10,92 & $-0,19$ & 11,23 & $-0,17$ & 10,87 & $-0,42$ & 11,46 & $-0,15$ \\
\hline L06xL05 & 12,42 & $-0,53$ & 12,84 & 0,31 & 10,55 & 0,11 & 8,58 & $-0,40$ & 12,75 & 0,56 & 11,43 & 0,01 \\
\hline HD2xL05 & 12,61 & 0,09 & 11,69 & $-0,36$ & 10,34 & $-0,49$ & 10,88 & 0,32 & 11,60 & $-0,22$ & 11,42 & $-0,13$ \\
\hline HD1xLF & 12,81 & 0,27 & 11,21 & 0,44 & 11,13 & 0,30 & 10,22 & 0,12 & 11,26 & 0,31 & 11,33 & 0,29 \\
\hline L10xL05 & 13,36 & 0,96 & 11,60 & 0,10 & 10,31 & 0,09 & 9,93 & 0,27 & 11,21 & 0,07 & 11,28 & 0,30 \\
\hline HT1xLF & 12,88 & 0,32 & 11,05 & 0,05 & 10,76 & $-0,22$ & 10,15 & $-0,05$ & 11,33 & $-0,27$ & 11,24 & $-0,03$ \\
\hline HS4xL05 & 12,70 & 0,22 & 11,44 & $-0,13$ & 10,23 & $-0,20$ & 10,39 & $-0,15$ & 11,19 & $-0,02$ & 11,19 & $-0,05$ \\
\hline Pob.36xL05 & 12,12 & $-0,36$ & 12,13 & $-0,04$ & 10,87 & 0,61 & 9,15 & 0,24 & 11,64 & 0,48 & 11,18 & 0,19 \\
\hline CMS 14-CxL05 & 12,85 & 0,24 & 11,41 & $-0,11$ & 10,78 & 0,15 & 8,95 & $-0,30$ & 11,74 & 0,15 & 11,14 & 0,02 \\
\hline HS3xL05 & 12,29 & $-0,52$ & 11,29 & $-0,19$ & 10,17 & 0,19 & 10,04 & $-0,07$ & 11,43 & $-0,09$ & 11,04 & $-0,14$ \\
\hline HD1xL05 & 12,54 & 0,51 & 10,93 & $-0,15$ & 10,58 & 0,43 & 9,80 & 0,57 & 11,26 & 0,40 & 11,02 & 0,35 \\
\hline Pob.36xLF & 12,57 & $-0,43$ & 12,02 & 0,17 & 10,29 & $-0,65$ & 9,60 & $-0,17$ & 10,59 & $-0,64$ & 11,01 & $-0,35$ \\
\hline HT3xL09 & 12,65 & 0,33 & 10,70 & $-0,28$ & 10,75 & $-0,01$ & 10,25 & 0,70 & 10,31 & 0,53 & 10,93 & 0,25 \\
\hline CMS 14-CxLF & 12,69 & $-0,43$ & 10,74 & $-0,47$ & 10,52 & $-0,79$ & 9,58 & $-0,54$ & 10,80 & $-0,87$ & 10,87 & $-0,62$ \\
\hline HT1xL05 & 11,68 & $-0,38$ & 11,15 & $-0,17$ & 10,50 & 0,21 & 9,52 & 0,19 & 11,47 & $-0,06$ & 10,86 & $-0,04$ \\
\hline HS1xL09 & 12,75 & 0,64 & 10,57 & $-0,38$ & 10,55 & $-0,22$ & 9,69 & $-0,09$ & 10,42 & 0,33 & 10,80 & 0,06 \\
\hline HD2xL09 & 11,89 & $-0,14$ & 11,24 & 0,57 & 10,92 & 0,63 & 9,98 & 0,14 & 9,63 & $-0,06$ & 10,73 & 0,23 \\
\hline CMS 14-CxL09 & 12,31 & 0,19 & 10,74 & 0,58 & 10,74 & 0,64 & 9,37 & 0,85 & 10,18 & 0,72 & 10,67 & 0,60 \\
\hline L06xL09 & 12,80 & 0,34 & 11,06 & $-0,10$ & 10,33 & 0,43 & 8,80 & 0,55 & 9,90 & $-0,17$ & 10,58 & 0,21 \\
\hline HT4xL05 & 12,32 & $-0,05$ & 11,58 & $-0,06$ & 9,16 & $-0,85$ & 9,18 & $-0,32$ & 10,48 & $-0,47$ & 10,54 & $-0,35$ \\
\hline HS $2 x L 09$ & 13,40 & 0,47 & 10,47 & $-0,19$ & 10,44 & $-0,06$ & 9,15 & $-0,31$ & 9,17 & $-0,16$ & 10,53 & $-0,05$ \\
\hline HS5xL09 & 11,78 & $-0,62$ & 11,07 & $-0,09$ & 10,05 & $-0,11$ & 9,63 & 0,22 & 10,00 & $-0,15$ & 10,51 & $-0,15$ \\
\hline HT2xL09 & 12,20 & $-0,38$ & 11,25 & 0,75 & 9,79 & $-0,06$ & 9,45 & 0,07 & 9,65 & 0,00 & 10,47 & 0,08 \\
\hline HS4xL09 & 11,80 & $-0,19$ & 10,24 & 0,05 & 10,27 & 0,39 & 10,12 & 0,32 & 9,53 & 0,44 & 10,39 & 0,20 \\
\hline Pob.36xL09 & 12,79 & 0,79 & 10,67 & $-0,12$ & 9,77 & 0,04 & 8,11 & $-0,07$ & 9,19 & 0,16 & 10,10 & 0,16 \\
\hline HS3xL09 & 12,18 & $-0,13$ & 10,21 & 0,11 & 9,45 & 0,02 & 8,86 & $-0,51$ & 9,30 & $-0,10$ & 10,00 & $-0,12$ \\
\hline HT1xL09 & 11,62 & 0,06 & 10,06 & 0,12 & 9,77 & 0,01 & 8,47 & $-0,14$ & 9,72 & 0,33 & 9,93 & 0,08 \\
\hline HT4xL09 & 11,87 & $-0,01$ & 10,02 & $-0,25$ & 9,30 & $-0,18$ & 8,45 & $-0,32$ & 8,53 & $-0,29$ & 9,63 & $-0,21$ \\
\hline L10xL09 & 11,34 & $-0,57$ & 9,65 & $-0,47$ & 8,88 & $-0,80$ & 8,23 & $-0,71$ & 8,16 & $-0,86$ & 9,25 & $-0,68$ \\
\hline HD1xL09 & 10,75 & $-0,78$ & 9,42 & $-0,29$ & 8,88 & $-0,73$ & 7,80 & $-0,69$ & 8,02 & $-0,71$ & 8,97 & $-0,64$ \\
\hline Pob. $36(\mathrm{~T})^{(2)}$ & 11,79 & - & 9,45 & - & 8,69 & - & 8,28 & - & 9,03 & - & 9,45 & - \\
\hline CMS 14-C (T) & 11,45 & - & 9,06 & - & 8,53 & - & 6,44 & - & 9,58 & - & 9,01 & - \\
\hline $\mathrm{Z} 8392(\mathrm{~T})$ & 12,47 & - & 10,73 & - & 10,16 & - & 10,52 & - & 10,86 & - & 10,95 & - \\
\hline P3071 (T) & 12,38 & - & 11,28 & - & 10,00 & - & 9,49 & - & 10,42 & - & 10,71 & - \\
\hline "Topcrosses" & 12,64 & - & 11,30 & - & 10,58 & - & 9,87 & - & 10,89 & - & 11,06 & - \\
\hline Testemunhas & 12,02 & - & 10,13 & - & 9,34 & - & 8,68 & - & 9,97 & - & 10,03 & - \\
\hline Média Geral & 12,59 & - & 11,21 & - & 10,48 & - & 9,77 & - & 10,82 & - & 10,97 & - \\
\hline $\mathrm{CV}(\%)$ & 4,39 & - & 5,02 & - & 6,66 & - & 6,56 & - & 7,90 & - & - & - \\
\hline $\mathrm{DMS}^{(3)}$ & 1,07 & - & 1,09 & - & 1,36 & - & 1,25 & - & 1,66 & - & - & - \\
\hline
\end{tabular}

${ }^{(1)} \mathrm{A} 1, \mathrm{~A} 2, \mathrm{~A} 3$, A4 e A5, correspondem aos ambientes de Londrina (PR), Palotina (PR), Xanxerê (SC) épocas 1 e 2 e Pinhão (PR), respectivamente.

${ }^{(2)}$ T: testemunha. ${ }^{(3)}$ Diferença mínima significativa pelo teste de Tukey a $5 \%$ de probabilidade. 
reduzir a amplitude da expressão das progênies e promover uma classificação errônea do mérito relativo de cada cruzamento (Hallauer \& Miranda Filho, 1995).

Quanto ao caráter PGC, as maiores estimativas de CGC I foram obtidas pelos parentais HS2 (0,79 t ha-1), HS5 (0,68 tha-1), HS1 e HT3 $\left(0,78 \mathrm{t} \mathrm{ha}^{-1}\right), \operatorname{HD} 2\left(0,75 \mathrm{t} \mathrm{ha}^{-1}\right)$ e HS5 $\left(0,71 \mathrm{t} \mathrm{ha}^{-1}\right)$, nos ambientes A1, A2, A3, A4 e A5, respectivamente (Tabela 4). A existência da interação ambiente $x$ CGC I e ambiente x CGC II, revelada na análise conjunta (Tabela 2), parece ter sido provocada predominantemente pela interação do tipo simples. Este fato pode ser observado com os parentais HS1, HS5 e HT3, que manifestaram efeitos positivos de CGC I em todos os ambientes, com exceção de HS1 com estimativa praticamente nula no ambiente A1, ao passo que os parentais L10, HT1, HT4 e HD1 mantiveram efeitos negativos. Quanto aos caracteres AP e AE, estas estimativas parecem ser ainda mais estáveis, tornando estas observações mais evidentes.

A análise de CGC II para o caráter PGC revela que a linhagem elite LF se destacou nos ambientes $\mathrm{A} 1, \mathrm{~A} 3$, A4 e A5, enquanto no ambiente A2, a melhor estimativa foi obtida pela linhagem L05. A linhagem L09 obteve as menores estimativas de
CGC (-0,50 t ha-1 a -1,44 tha-1). Estes resultados demonstram que a maior frequiência de alelos favoráveis para aumentar o caráter PGC se encontra no testador LF, enquanto a menor freqüência encontrase no L09.

Em relação aos caracteres AP e AE, valores negativos de CGC I são desejáveis e foram obtidos pelo parental HT1 no ambiente A1 e parental L06 nos ambientes A2, A3, A4 e A5. As estimativas de CGC II mais favoráveis foram obtidas pelo testador L09, variando para AP de $-13,10 \mathrm{~cm}$ a $-19,22 \mathrm{~cm} \mathrm{e}$ para AE de $-11,78 \mathrm{~cm}$ a $-13,70 \mathrm{~cm}$. No ambiente A4, em que se caracterizaram as maiores estimativas de $\mathrm{AP}$ e AE, esta linhagem intensificou o seu efeito redutor, com uma estimativa média de $-19,22 \mathrm{~cm}$ para AP e $-13,70 \mathrm{~cm}$ para AE.

Em todos os ambientes, a linhagem elite LF apresentou efeitos de CGC que contribuíram para aumentar os caracteres PGC, AP e AE e a L09 apresentou efeitos de CGC para reduzir estes caracteres. O testador L05 apresentou efeitos variáveis de CGC para reduzir ou aumentar PGC e favoráveis para aumentar AP e AE. As diferenças nas freqüências alélicas apresentadas pelos testadores repercutiram na capacidade discriminatória exercida sobre os parentais, concordando com os trabalhos de Keller

Tabela 4. Estimativas dos efeitos de capacidade geral de combinação, associados ao material parental (CGC I) e às linhagens elites testadoras (CGC II), nos caracteres peso de grãos corrigido (PGC, em tha ${ }^{-1}$ ), altura de planta (AP, em cm) e altura de espiga (AE, em cm), em cinco ambientes (A), 2000/2001(1).

\begin{tabular}{|c|c|c|c|c|c|c|c|c|c|c|c|c|c|c|c|}
\hline \multirow[t]{2}{*}{ Germoplasma } & \multicolumn{5}{|c|}{ PGC } & \multicolumn{5}{|c|}{ AP } & \multicolumn{5}{|c|}{$\mathrm{AE}$} \\
\hline & $\mathrm{A} 1$ & A2 & A3 & A4 & A5 & A1 & A2 & A3 & A4 & A5 & A1 & A2 & A3 & A4 & A5 \\
\hline & & & & & & & & CGC I & & & & & & & \\
\hline L06 & 0,32 & 0,67 & $-0,10$ & $-0,85$ & 0,62 & $-10,00$ & $-9,67$ & $-13,49$ & $-17,35$ & $-8,05$ & $-2,99$ & $-7,26$ & $-12,42$ & $-11,81$ & $-5,80$ \\
\hline L10 & $-0,23$ & $-0,37$ & $-0,31$ & $-0,15$ & $-0,42$ & $-1,98$ & $-4,49$ & $-5,50$ & $-1,50$ & $-3,66$ & 0,76 & 0,02 & $-1,66$ & $-0,68$ & $-4,78$ \\
\hline HS1 & $-0,03$ & 0,46 & 0,78 & 0,69 & 0,64 & 4,76 & 3,25 & 7,35 & 9,32 & 1,68 & 3,00 & 5,02 & 7,99 & 8,30 & 4,26 \\
\hline HS2 & 0,79 & 0,17 & 0,50 & 0,37 & $-0,12$ & 0,41 & $-4,38$ & $-2,32$ & $-0,02$ & $-2,45$ & 5,67 & $-1,83$ & $-2,95$ & 2,42 & $-1,63$ \\
\hline HS3 & 0,18 & $-0,39$ & $-0,56$ & 0,28 & $-0,04$ & 0,80 & 2,25 & 3,94 & 3,72 & $-0,27$ & $-7,05$ & $-4,61$ & $-2,56$ & $-3,46$ & $-2,80$ \\
\hline HS4 & $-0,16$ & $-0,30$ & $-0,11$ & 0,71 & $-0,36$ & $-7,48$ & $-5,39$ & $-3,89$ & $-4,50$ & $-3,49$ & $-5,98$ & $-4,59$ & $-5,16$ & $-4,57$ & $-2,37$ \\
\hline HS5 & 0,26 & 0,68 & 0,17 & 0,32 & 0,71 & 2,48 & 4,87 & 1,70 & 3,47 & 2,77 & 0,12 & 4,22 & $-0,12$ & 2,03 & 0,53 \\
\hline HT1 & $-0,58$ & $-0,55$ & $-0,24$ & $-0,49$ & $-0,05$ & $-12,09$ & $-7,58$ & $-4,23$ & $-8,00$ & $-4,94$ & $-8,38$ & $-5,46$ & $-2,40$ & $-5,44$ & $-4,76$ \\
\hline HT2 & 0,44 & 0,00 & $-0,14$ & 0,29 & 0,20 & 1,96 & 1,90 & 6,81 & 4,11 & 5,58 & $-0,46$ & 0,80 & 2,44 & 1,84 & 2,59 \\
\hline HT3 & 0,18 & 0,49 & 0,78 & 0,46 & 0,33 & 2,37 & 2,07 & 3,72 & $-2,77$ & 1,86 & 1,91 & $-1,30$ & 2,95 & $-1,61$ & 2,68 \\
\hline HT4 & $-0,26$ & $-0,23$ & $-0,52$ & $-0,32$ & $-0,63$ & $-2,94$ & 3,57 & 1,38 & $-2,11$ & 1,12 & $-2,61$ & $-0,33$ & 2,88 & $-2,44$ & 2,22 \\
\hline HD1 & $-0,61$ & $-0,78$ & $-0,38$ & $-0,59$ & $-0,71$ & $-0,59$ & $-2,30$ & $-4,56$ & $-4,27$ & $-4,01$ & 1,86 & 3,96 & $-1,08$ & $-0,40$ & 0,59 \\
\hline HD2 & $-0,12$ & 0,18 & 0,30 & 0,75 & 0,24 & 1,89 & 1,11 & 0,88 & 3,74 & 3,67 & $-1,74$ & $-0,65$ & 0,09 & 1,51 & 1,22 \\
\hline Pob. 36 & $-0,15$ & 0,30 & $-0,27$ & $-0,91$ & $-0,42$ & 5,69 & 6,24 & 1,33 & 1,17 & 0,62 & 4,41 & 4,89 & 3,84 & 2,40 & 0,59 \\
\hline \multirow[t]{2}{*}{ CMS 14-C } & $-0,03$ & $-0,34$ & 0,10 & $-0,57$ & 0,02 & 14,74 & 8,55 & 6,88 & 14,98 & 9,58 & 11,47 & 7,11 & 8,16 & 11,90 & 7,48 \\
\hline & \multicolumn{15}{|c|}{ CGC II } \\
\hline LF & 0,51 & 0,25 & 0,64 & 0,82 & 0,76 & 8,35 & 8,65 & 9,38 & 13,80 & 6,62 & 6,13 & 6,99 & 8,75 & 8,57 & 5,69 \\
\hline L09 & $-0,50$ & $-0,81$ & $-0,59$ & $-0,78$ & $-1,44$ & $-13,10$ & $-13,29$ & $-14,79$ & $-19,22$ & $-13,53$ & $-11,88$ & $-11,78$ & $-13,39$ & $-13,70$ & $-11,84$ \\
\hline L05 & $-0,01$ & 0,56 & $-0,05$ & $-0,05$ & 0,68 & 4,75 & 4,63 & 5,41 & 5,42 & 6,90 & 5,75 & 4,79 & 4,64 & 5,12 & 6,15 \\
\hline
\end{tabular}


(1949) e Lonnquist \& Lindsey (1964), revelando que a melhor discriminação entre "topcrosses" foi promovida pelos testadores que apresentam uma baixa freqüência de alelos favoráveis (Vencovsky \& Barriga, 1992; Hallauer \& Miranda Filho, 1995; Elias et al., 2000). Desta forma, a seleção de uma ou outra linhagem avaliadora irá depender dos objetivos de cada programa de melhoramento. Para empresas produtoras de sementes de milho híbrido, o testador ideal é aquele que possui um alto rendimento per se, oferecendo uma discriminação adequada da base genética avaliada e formando boas combinações híbridas. Considerando estes atributos, a linhagem LF pode ser definida como o melhor dos três testadores avaliados.

As análises de CEC estão relacionadas com a distância genética entre os progenitores e evidenciam a importância de interações não aditivas, caracterizadas pela complementaridade em relação à frequiência dos alelos nos locos com alguma dominância entre os genitores. Altos valores positivos ou negativos indicam que o desempenho de algumas combinações híbridas são relativamente superiores ou inferiores ao que seria esperado com base na média dos parentais envolvidos (Sprague \& Tatum, 1942).

A ausência de significância da interação ambiente x CEC, verificada na análise conjunta de variância (Tabela 2), revela que os cruzamentos apresentaram comportamento estável nos diferentes ambientes, sendo possível utilizar a média de CEC como parâmetro de seleção de cruzamentos específicos. Para PGC, as estimativas médias de CEC variaram de $-0,68 \mathrm{t} \mathrm{ha}^{-1}$ a $0,60 \mathrm{t} \mathrm{ha}^{-1}$ (Tabela 3 ). As cinco maiores médias positivas foram obtidas pelas combinações CMS 14-C x L09, HT4 x LF, L10 x LF, HD1 x L05, L10 x L05 e, destas, apenas os cruzamentos que envolveram a participação do testador LF estão entre as 10 melhores médias gerais. Os parentais HS3, HS5 e HT4 apresentam as melhores estimativas de CEC quando cruzados com a linha LF enquanto os parentais CMS 14-C, HD2, HS4, HT3 e L06, quando foram cruzados com a linhagem L09. Os parentais HD1 e L10 apresentam estimativas de CEC favoráveis nas combinações envolvendo as linhagens LF e L05, enquanto o parental Pob. 36, nas combinações com L05 e L09. Os parentais HS1, HS2, HT1 e HT2 apresentaram estimativas de CEC praticamente nulas, independentemente do testador utilizado.

\section{Conclusões}

1. Os testadores promovem uma classificação diferenciada para a base genética estudada.

2. A linhagem LF estabelece as melhores combinações híbridas, mas nem sempre proporciona a melhor discriminação entre os cruzamentos.

3. Nos ambientes avaliados, nenhum dos testadores apresenta a capacidade de melhorar simultaneamente todas as características observadas, em cruzamentos com os quinze parentais.

4. Entre as combinações híbridas de alta performance para todos os caracteres, destacam-se os cruzamentos entre os parentais HS5, HS1, HS2 e HT4 com a linhagem LF e os parentais HS5 e L10 com L05.

\section{Agradecimentos}

Ao Dr. Antônio Carlos Gerage (Iapar), à empresa Agroeste Sementes Ltda, pela colaboração na realização do trabalho; à Capes, pela concessão da bolsa de estudo.

\section{Referências}

CASTELLANOS, J. S.; HALLAUER, A. R.; CORDOVA, H. S. Relative performance of testers to identify elite lines of corn (Zea mays L.). Maydica, Bergamo, v. 43, n. 3, p. 217-226, 1998.

DUARTE, A. P.; PATERNIANI, M. E. A. G. Z. (Coord.). Cultivares de milho no Estado de São Paulo. Campinas: Instituto Agronômico, 1998. 81 p. (Documento IAC, 62).

DUDLEY, J. W.; SAGHAI MAROOF, M. A.; RUFENER, G. K. Molecular marker information and selection of parents in corn breeding programs. Crop Science, Madison, v. 32, p. 301-304, 1992.

ELIAS, H. T.; CARVALHO, S. P.; ANDRÉ, C. G. M. Comparação de testadores na avaliação de famílias $S_{2}$ de milho. Pesquisa Agropecuária Brasileira, Brasília, v. 35, n. 6 , p. 1135-1142, jun. 2000. 
EVERETT, L. A.; ETA-NDU, J. T.; NDIORO, M.; WALKER, P. Combining ability among source populations for tropical mid-altitude maize inbreeds. Maydica, Bergamo, v. 40, p. 165-171, 1995.

EYHÉRABIDE, G. H.; GONZALEZ, A. S. Interactions between testers and Argentine maize landraces. Maydica, Bergamo, v. 42, p. 29-38, 1997.

FERREIRA, D. F.; OLIVEIRA, A. C.; SANTOS, M. X.; RAMALHO, M. A. P. Métodos de avaliação da divergência genética em milho e suas relações com os cruzamentos dialélicos. Pesquisa Agropecuária Brasileira, Brasília, v. 30, n. 9, p. 1189-1194, set. 1995.

GRIFFING, B. Concept of general and specific combining ability in relation to diallel crossing systems. Australian Journal of Biological Sciences, Collingwood, v. 9, p. 463493, 1956.

HALLAUER, A. R.; MIRANDA FILHO, J. B. de. Quantitative genetics in maize breeding. 2nd ed. Ames: Iowa State University Press, 1995. 468 p.

KELLER, K. R. A comparison involving the number of, and relationship between, testers in evaluating inbred lines of maize. Agronomy Journal, Madison, v. 41, p. 323-331, 1949.

LONNQUIST, J. H.; LINDSEY, M. F. Topcross versus $\mathrm{S}_{1}$ line performance in corn (Zea mays L.). Crop Science, Madison, v. 4, p. 580-584, 1964.

MAGNAVACA, R. Desenvolvimento de híbridos em empresas privadas. In: SIMPÓSIO SOBRE ATUALIZAÇÃO EM GENÉTICA E MELHORAMENTO DE PLANTAS, 2., 1998, Lavras. Anais... Lavras: Editora Ufla, 1998. p. $163-170$.

MIRANDA FILHO, J. B. de; VENCOVSKY, R. Analysis of variance with interaction of effects. Brazilian Journal of Genetics, Ribeirão Preto, v. 18, n. 1, p. 129-134, 1995.

NASS, L. L.; LIMA, M.; VENCOVSKY, R.; GALLO, P. B. Combining ability of maize inbred lines evaluated in three environments in Brazil. Scientia Agricola, Piracicaba, v. 57, n. 1, p. 129-134, 2000.

PINTO, R. M. C.; GARCIA, A. A. F.; SOUZA JÚNIOR, C. L. Alocação de linhagens de milho derivadas das popu- lações BR-105 e BR-106 em grupos heteróticos. Scientia Agricola, Piracicaba, v. 58, n. 3, p. 541-548, 2001.

RADOVIC, G.; JELOVAC, D. Identification of the heterotic pattern in Yugoslav maize germplasm. Maydica, Bergamo, v. 40, p. 223-227, 1995.

RAMALHO, M. A. P.; SANTOS, J. B.; ZIMMERMANN, M. J. de O. Interação dos genótipos x ambientes. In: RAMALHO, M. A. P.; SANTOS, J. B.; ZIMMERMANN, $\mathrm{M}$. J. de $\mathrm{O}$. Genética quantitativa em plantas autógamas: aplicação no melhoramento do feijoeiro. Goiânia: Editora UFG, 1993. p. 131-169.

SANTOS, M. X.; POLLAK, L. M.; CARVALHO, H. W. L.; PACHECO, C. A. P.; GAMA, E. E. G.; GUIMARÃES, P. E. de O.; ANDRADE, R. V. Heterotic responses of tropical elite maize accessions from Latin America with Brazilian testers. Scientia Agricola, Piracicaba, v. 58, n. 4, p. 767-775, 2001.

SAN VICENTE, F. M.; BEJARANO, A.; MARIN, C.; CROSSA, J. Analysis of diallel crosses among improved tropical white endosperm maize populations. Maydica, Bergamo, v. 43, p. 147-153, 1998.

SINOBAS, J.; MONTEAGUDO, I. Heterotic patterns among U. S. corn belt and Spanish maize populations. Maydica, Bergamo, v. 41, p. 143-148, 1996.

SPRAGUE, G. F.; TATUM, L. A. General vs specific combining ability in single crosses of corn. Journal of the American Society of Agronomy, Madison, v. 34, p. $923-932,1942$.

VASAL, S. K.; SRINIVASAN, G.; CROSSA J.; BECK, D. L. Heterosis and combining ability of CIMMYT's subtropical and temperate early-maturity maize germplasm. Crop Science, Madison, v. 32, p. 884-890, 1992a.

VASAL, S. K.; SRINIVASAN, G.; GONZALEZ, F.; PANDEY, S.; BECK, D. L.; CROSSA, J. Heterosis and combining ability of CIMMYT's tropical x subtropical maize germplasm. Crop Science, Madison, v. 32, p. 1483 1489, 1992 b.

VENCOVSKY, R.; BARRIGA, P. Genética biométrica no fitomelhoramento. Ribeirão Preto: Sociedade Brasileira de Genética, 1992. 496 p. 